\title{
Vasopressin Reduces Cardiac Function and Augments Cardiopulmonary Baroreflex Resistance Increases in Man
}

Thomas J. Ebert, Allen W. Cowley, Jr., and Meredith Skelton

Department of Physiology, The Medical College of Wisconsin, and the Veterans Administration Medical Center, Milwaukee, Wisconsin 53226

\begin{abstract}
We examined the effects of physiologic infusions of arginine vasopressin (AVP) on cardiòvascular hemodynamics and on reflex responses initiated by decreasing cardiopulmonary baroreceptor stimulation (with lower body negative pressure) in $\mathbf{1 0}$ healthy, captopril-pretreated young men (19-27 yr). Their responses were compared with those of four volunteers given isosmotic infusion. Heart rate, stroke volume, blood pressure, and forearm blood flow were measured by electrocardiography, impedance cardiography, radial artery cannulation, and strain gauge plethysmography. Two 55-min infusions of AVP at rates of 0.15 and $0.40 \mathrm{ng} / \mathrm{kg}$ per min increased average plasma concentrations from control levels of $5 \mathrm{pg} / \mathrm{ml}$ to 18 and $36 \mathrm{pg} / \mathrm{ml}$, respectively. These infusions resulted in progressive reductions of heart rate and cardiac output and increases of forearm and total peripheral resistance. Blood pressure increases were significant only during the larger AVP infusion rate. Lower body negative pressure provoked reflex increases of total peripheral resistance. These increases were enhanced 60\% during AVP infusion compared with increases during control (pre-AVP). Baseline measurements and reflex responses were unchanged by isosmotic infusions. These results demonstrate that AVP has profound effects on cardiovascular function and augments cardiopulmonary baroreflex-mediated increases of peripheral resistance in man.
\end{abstract}

\section{Introduction}

Studies from experimental animals demonstrate that elevations of plasma arginine vasopressin (AVP) ${ }^{1}$ levels within the physiologic range have profound effects on cardiovascular function and autonomic reflexes (1-5). There are very few reports of the cardiovascular effects of AVP infusion in man. Available evidence indicates that small increases of plasma AVP levels result in decreases of heart rate but do not change blood pressure (68). When AVP is infused into patients with autonomic dysfunction or into normal subjects after ganglionic blockade, heart

Please address correspondence to Dr. Ebert, Department of Physiology, Medical College of Wisconsin, 8701 West Watertown Plank Rd., Milwaukee, WI 53226.

Received for publication 29 January 1985 and in revised form 3 December 1985 .

1. Abbreviations used in this paper: AVP, arginine vasopressin; D5W, isosmotic; ECG, electrocardiograph; lbnp, lower body negative pressure; Zo, transthoracic impedance.

J. Clin. Invest.

(C) The American Society for Clinical Investigation, Inc.

0021-9738/86/04/1136/07 \$1.00

Volume 77, April 1986, 1136-1142 rate does not decrease, but blood pressure increases substantially $(7,8)$. These data suggest that the pressor effects of AVP are buffered by a neurally mediated bradycardia and a decline in cardiac output.

The influence of AVP on cardiac output and total peripheral resistance in healthy man is not known. Despite numerous reports of the effect of AVP on cardiovascular function in experimental animals, we were not confident that similar effects occurred in conscious man since human autonomic neural control mechanisms differ from those of other species $(9,10)$. Therefore, one objective of this investigation was to determine the effects of increasing plasma AVP levels, within a physiologic range, on cardiac output and peripheral resistance in healthy man.

A second objective was to test the hypothesis that AVP alters human cardiopulmonary baroreflex control of peripheral resistance. This inquiry was prompted by previous data which have shown that AVP has both a direct effect of constricting vascular smooth muscle $(11,12)$ and a major influence on autonomic neural mechanisms in animals (2-5). Cardiopulmonary baroreceptors respond to slight alterations in central blood volume and initiate reflex changes in peripheral resistance to maintain a stable blood pressure $(13,14)$. If AVP is released during hemorrhage or orthostasis $(15,16)$, it may have a profound effect on reflex resistance increases initiated by low-pressure baroreceptors.

\section{Methods}

These studies were approved by both institutions' Human Research Review Committee. 14 healthy normotensive adults were studied after they gave written consent to participate. Their average age $( \pm S E M)$ was $21 \pm 3$ yr. 10 subjects received intravenous infusions of AVP (Pitressin, ParkeDavis, Inc., Morris Plains, NJ) and four served as time controls and received intravenous infusion of isosmotic (D5W) solution. The total volume of infusion was similar in each group. Studies were conducted with subjects lying supine in a quiet warm $\left(23-25^{\circ} \mathrm{C}\right)$ room.

Measurements. Heart rate was measured from the electrocardiograph (ECG). Blood pressure was monitored with a Gould P50 transducer (Gould, Inc., Hato Rey, Puerto Rico) connected to a 20-gauge catheter inserted into the radial artery. The analogue signal was recorded on a strip chart recorder and amplified so that digital conversion of pressure recordings were accurate to $0.5 \mathrm{mmHg}$. Mean arterial pressure was calculated as diastolic pressure plus one-third of the pulse pressure. Forearm blood flow was obtained from a temperature compensated $\mathrm{Hg}$-in-Silastic strain gauge on the forearm. A blood pressure cuff was placed about the upper arm for intermittent venous occlusion and an infant's cuff placed at the wrist for arterial occlusion. This method is described in detail elsewhere (17). Analogue data were transcribed on an ink-writing recorder. Digital values were obtained by manual cursor placement on a digitizing board on-line with a digital computer.

Impedance cardiography. A tetrapolar impedance cardiograph (Surcom, Inc., Minneapolis, MN) was used to determine stroke volume. Three electrodes were positioned circumferentially about the body: at the lower neck, xiphoid process, and $8 \mathrm{~cm}$ below the xiphoid process. A fourth electrode was placed on the forehead (18). A 100-kHz, 4-mA 
current was transmitted through the outer two electrodes and transthoracic impedance $(\mathrm{Zo})$ changes were detected from the inner two electrodes. The first derivative of the Zo change (associated with ventricular contraction) was used to calculate stroke volume $(18,19)$, and changes in baseline Zo were used to detect relative changes in intrathoracic fluid. It has been established in this laboratory $(18,19)$ and elsewhere that stroke volume and cardiac output, calculated from analogue-impedance signals, correlate well with those derived from invasive measurements obtained with Fick $(20,21)$, dye $(19,22-24)$, and thermal $(25,26)$ dilution methods. In these comparisons the correlations between changes of stroke volume have been stronger than those between absolute stroke volumes $(18,19,22-24)$. We have previously established a close correlation between beat-by-beat impedance and left ventricular (ventriculography) stroke volumes $(r=0.79)$ and stroke volume changes $(r=0.89)$ in patients undergoing cardiac catheterization (18). The impedance method detected accurately stroke volume changes ranging from 1 to $100 \mathrm{ml} /$ beat.

Total peripheral resistance was calculated from the ratio of impedance-derived cardiac output and arterial blood pressure. In this study, there was a significant correlation $(r=0.751, P<0.01)$ between baseline total peripheral resistance and plasma norepinephrine, an index of sympathetic activity (27). This strengthens our contention that impedancederived parameters are reliable.

Baseline Zo can be used as an index of thoracic blood volume. We reason that such estimates are accurate based upon the following: $(a)$ changes in Zo correlate closely with changes of central blood volume in animals (28-30); (b) in this lab, changes of calf blood volume (produced by intermittent graded venous occlusions of the upper thigh), which are detected by impedance, correlate well $(r=0.87)$ with estimates made with an Hg-in-Silastic strain gauge; and $(c) \mathrm{Zo}$ changes correlate closely $(r=0.989)$ with reductions in central venous pressure produced by graded lower body negative pressure (31).

Biochemical measurements. Blood samples were obtained from the arterial catheter and analyzed in the Bioassay Laboratory located in our Physiology Department. Norepinephrine and epinephrine were determined by radioenzymatic assay using the Cat-A-Kit (Upjohn Co., Kalamazoo, MI). Plasma renin activity was determined by radioimmunoassay (RIA) of the generation of angiotensin I using a modification of the technique developed by Sealy et al. (32). Plasma AVP concentration was measured using an RIA procedure developed in this laboratory and described previously in detail $(33,34)$. Plasma samples $(1 \mathrm{ml})$, which were deproteinized with $2 \mathrm{ml}$ acetone, were taken to complete dryness using a centrifugation-vacuum-concentrating system (Savant Instruments, Hicksville, NY) and assayed as reported previously. Protein precipitation of human plasma resulted in a 20-25\% loss determined with both ${ }^{125}$ I-AVP and AVP. Reported values were corrected for $23 \%$ extraction loss and therefore represent the concentrations in the original 1 -ml plasma sample. The between-assay variability based on 12 separate standard curves averaged $\pm 4.4 \%$. The intraassay coefficient of variation averaged $\pm 4.0 \%$. The least detectable AVP concentration in the original plasma averaged $0.3 \mathrm{pg} / \mathrm{ml}$, and the mid-range of the assay averaged 6.5 $\mathrm{pg} / \mathrm{ml}$.

Protocol. Subjects abstained from alcohol, caffeine, and nicotine for $24 \mathrm{~h}$ before each study and fasted from midnight to 7 a.m. on the day of the study. They ate a light breakfast in the laboratory which consisted of a doughnut and $250 \mathrm{ml}$ of orange juice. Volunteers were given $25 \mathrm{mg}$ of captopril (E. R. Squibb \& Sons, Princeton, NJ). This prevented fluctuations in angiotensin II which could have influenced responses to AVP infusion $(35,36)$. Intravenous and radial artery catheters were inserted after local infiltration with lidocaine. Impedance electrodes, ECG electrodes, heart sounds microphone, and forearm strain gauge and cuffs were placed. Volunteers were positioned in the lower body negative pressure (lbnp) box. A practice trial of $10 \mathrm{mmHg}$ of lbnp was initiated to familiarize each subject with this procedure. After a 45-min supine rest period, arterial blood was sampled for plasma renin activity, AVP, and catecholamines. This was followed by three brief (10-s), relaxed, endexpiratory breath holds (at 2-min intervals). Baseline (control) data were obtained from five cardiac cycles during each of these three periods of apnea and were averaged. This was followed by a 3-min period of lbnp (10 $\mathrm{mmHg}$ ) during which $3-10 \mathrm{~s}$ breath hold samples were obtained at 1 -min intervals and averaged. Low-dose intravenous AVP $(0.15 \mathrm{ng} / \mathrm{kg}$ per min) was infused for $55 \mathrm{~min}$ to assure steady state plasma levels. During the last $10 \mathrm{~min}$ of infusion, blood sampling, and baseline and lbnp measurements (during apnea) were repeated. This procedure was followed by a $55-\mathrm{min}$ infusion period of a higher dose of AVP ( $0.40 \mathrm{ng} /$ $\mathrm{kg}$ per min) and repeat control, lbnp, and blood samples were obtained.

Statistical analyses. Control data before each application of lbnp were averaged and compared with mean responses during lbnp with Student's $t$ tests for paired data. Responses of the time control group were compared with the experimental group with analyses of variance. Changes in hemodynamic parameters and lbnp responses during AVP infusion were compared with responses before AVP infusion with paired $t$ tests. Differences were assumed to be significant if $P$ values were $<0.05$. Data are presented as means \pm SEM.

\section{Results}

Plasma AVP, catecholamines, and renin activity are shown in Fig. 1. Baseline AVP levels averaged $5 \pm 1.1$ (mean \pm SEM) pg/ $\mathrm{ml}$ and increased to $18 \pm 1.3$ and $36 \pm 3.8 \mathrm{pg} / \mathrm{ml}$ during infusion of $0.15 \mathrm{ng} / \mathrm{kg}$ per min of AVP and during $0.40 \mathrm{ng} / \mathrm{kg}$ per min of AVP, respectively. Since volunteers were given captopril before the study, plasma renin activity was elevated from normal levels (which range between 1 and $2 \mathrm{ng} / \mathrm{ml}$ per $\mathrm{h}$ in supine men). Renin activity had begun to return toward baseline during the last $15 \mathrm{~min}$ of the study. Plasma epinephrine was unchanged throughout the testing protocol. However, norepinephrine tended to decrease at the low-dose AVP infusion and was significantly $(P<0.05)$ decreased from control during the larger dose of AVP. Plasma AVP (Fig. 2), renin activity, and catecholamines were unchanged in the time control group. Plasma renin activity $(\mathrm{ng} / \mathrm{ml}$ per $\mathrm{h})$ and norepinephrine $(\mathrm{pg} / \mathrm{ml})$ averaged $8.7 \pm 1.8$ and $205 \pm 18$, respectively, during control. These were $8.5 \pm 2.6$ and $216 \pm 18$ during the first placebo infusion and were $7.1 \pm 3.1$ and $222 \pm 21$ during the second placebo infusion.
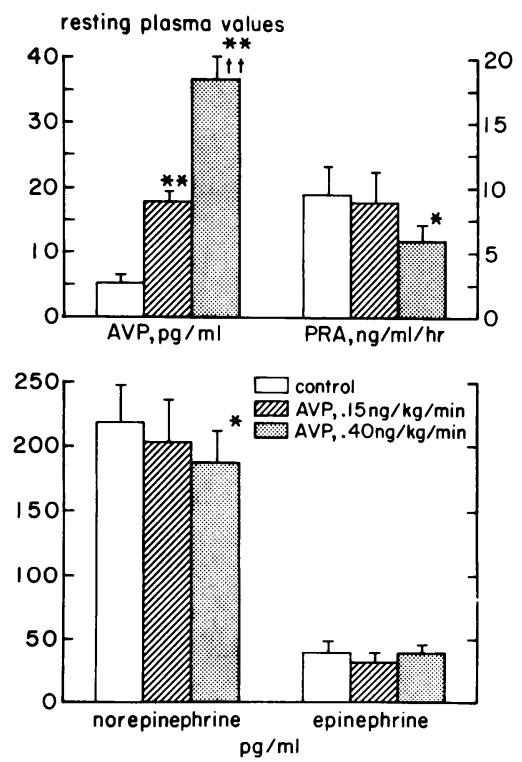

Figure 1. Plasma values measured before and during AVP infusion in 10 volunteers. PRA, plasma renin activity. ${ }^{*},{ }^{* *}$, significantly changed from control at $P<0.05$ and 0.01 levels. ++ , significantly changed from low-dose AVP infusions, $P<0.01$. 


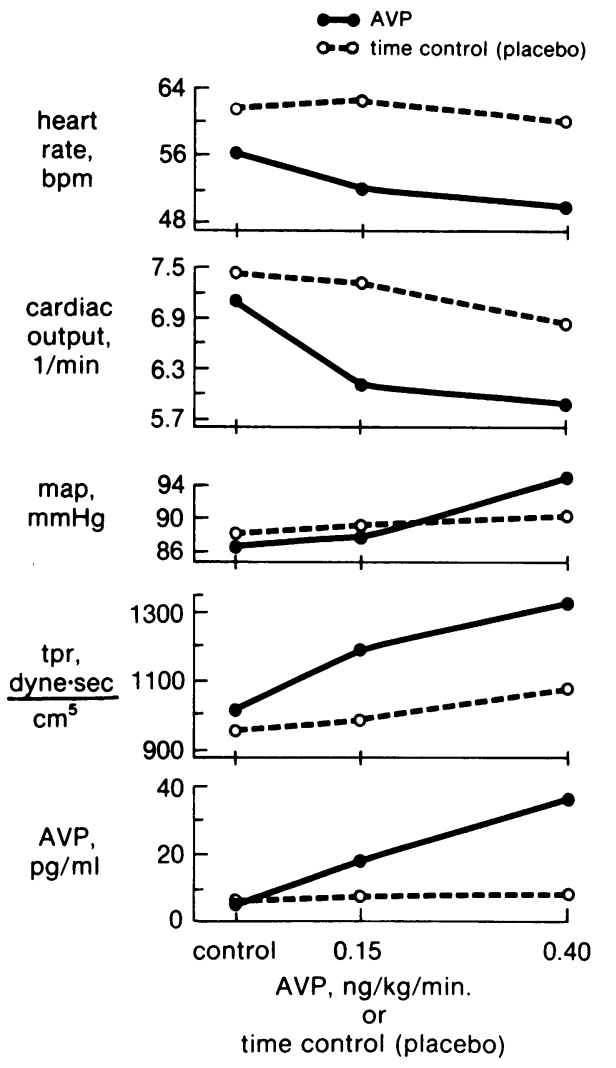

Figure 2. Cardiovascular responses to AVP $(n=10)$ or placebo ( $n$ $=4$ ). All responses to AVP infusion were significantly different from time controls except mean arterial pressure (map) responses to lowdose AVP infusion.

Resting hemodynamics. Baseline heart rate $(56 \pm 2.5$ beats per minute) declined significantly by $4 \pm 0.8$ and $6 \pm 1.2$ beats per minute with increasing AVP infusion (Fig. 3). Significant decreases were observed in stroke volume (8\%) and cardiac output (15\%) during AVP infusions. No distinctions could be made in the responses between the low and higher doses of AVP. During low-dose AVP infusions, there were no changes in systolic and mean arterial pressures, but there were slight $(2 \pm 0.6 \mathrm{mmHg})$ and significant increases in diastolic blood pressures from baseline. The higher AVP infusion rate produced small but significant increases of systolic $(3.6 \pm 0.8 \mathrm{mmHg})$, diastolic $(9.0 \pm 2.1 \mathrm{mmHg})$, and mean $(7.9 \pm 1.3 \mathrm{mmHg}$ ) arterial pressures (Fig. 3). Baseline total peripheral resistance $\left(1,018 \pm 96 \mathrm{dyne} \cdot \mathrm{sec} \cdot \mathrm{cm}^{-5}\right)$ increased progressively to $1,195 \pm 117$ and $1,333 \pm 104 \mathrm{dyne} \cdot \mathrm{sec} \cdot \mathrm{cm}^{-5}$ during low-dose and high-dose AVP infusion (Fig. 4). A similar and significant progressive increase of forearm vascular resistance was noted. This figure also demonstrates that $\mathrm{Zo}$, a reliable index of thoracic blood volume (31), was unchanged during AVP infusions.

Baseline hemodynamic parameters were minimally changed in the time control group, as shown in Fig. 2. Analysis of variance demonstrated a significant $(P<0.05)$ effect of AVP on all variables (except $\mathrm{Zo}$ ) when compared with the time controls.

Responses to low-level lbnp. Heart rate and arterial blood pressure were unchanged by lbnp both before and after AVP infusion (Fig. 5). However, stroke volume and cardiac output were decreased significantly by lbnp and to a comparable extent during control and during AVP infusion. In contrast, the reflex
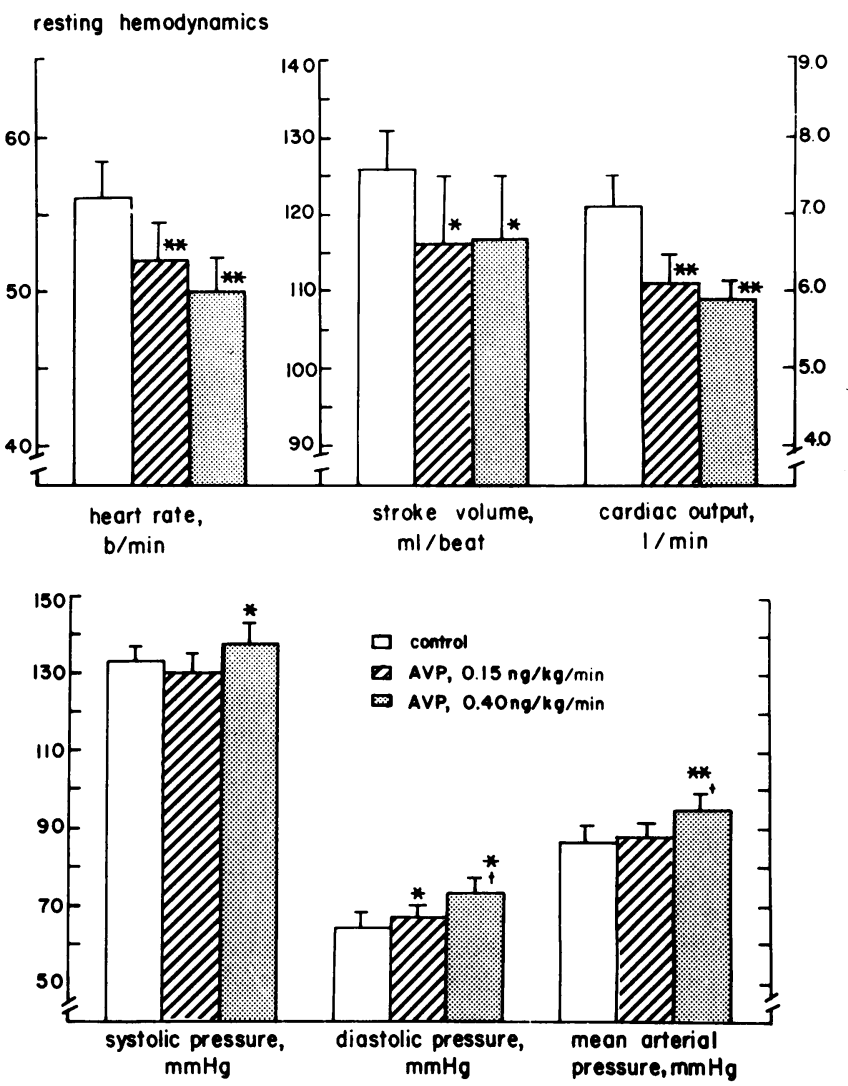

Figure 3. Cardiovascular responses to AVP infusion in 10 supine men. Control values are those obtained before AVP infusion. *, **, significant change from control, $P<0.05$ and 0.01 . + , significant change from low-dose AVP infusion, $P<0.05$.

increases of total peripheral resistance produced by lbnp $(96 \pm 15$ dyne $\cdot \mathrm{sec} \cdot \mathrm{cm}^{-5}$ ) were increased by $60 \%$ during low- and highdose AVP infusion (to $157 \pm 47$ and $155 \pm 44 \mathrm{dyne} \cdot \mathrm{sec} \cdot \mathrm{cm}^{-5}$, respectively; Fig. 6). AVP did not have a similar enhancing effect on reflex increases of forearm vascular resistance.

Zo was used to monitor the decreases in thoracic blood volume produced by lbnp. As shown in Fig. 6, the Zo changes were

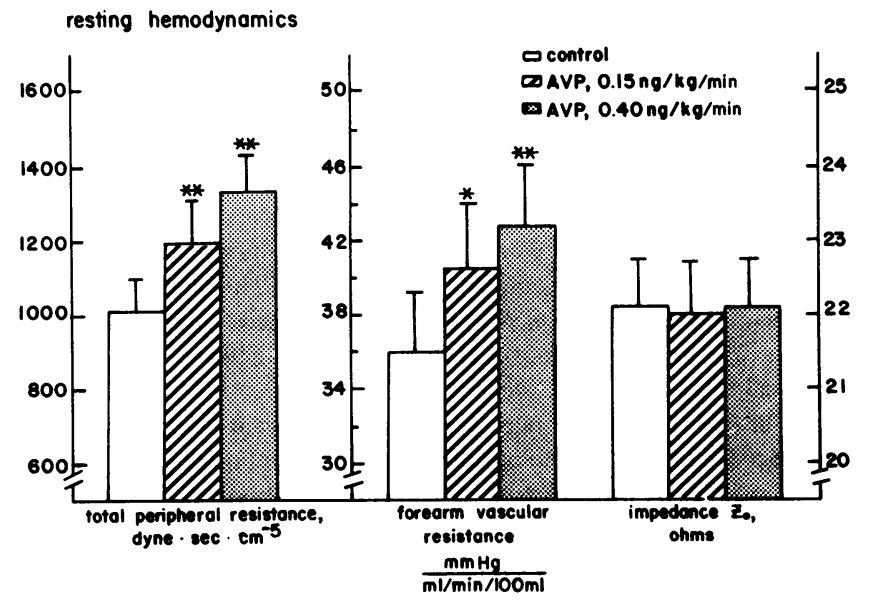

Figure 4. Resistance and Zo responses to AVP infusion. Key as in Fig. 2. $\mathrm{Zo}_{\mathrm{o}}$ is an index of thoracic blood volume changes. 

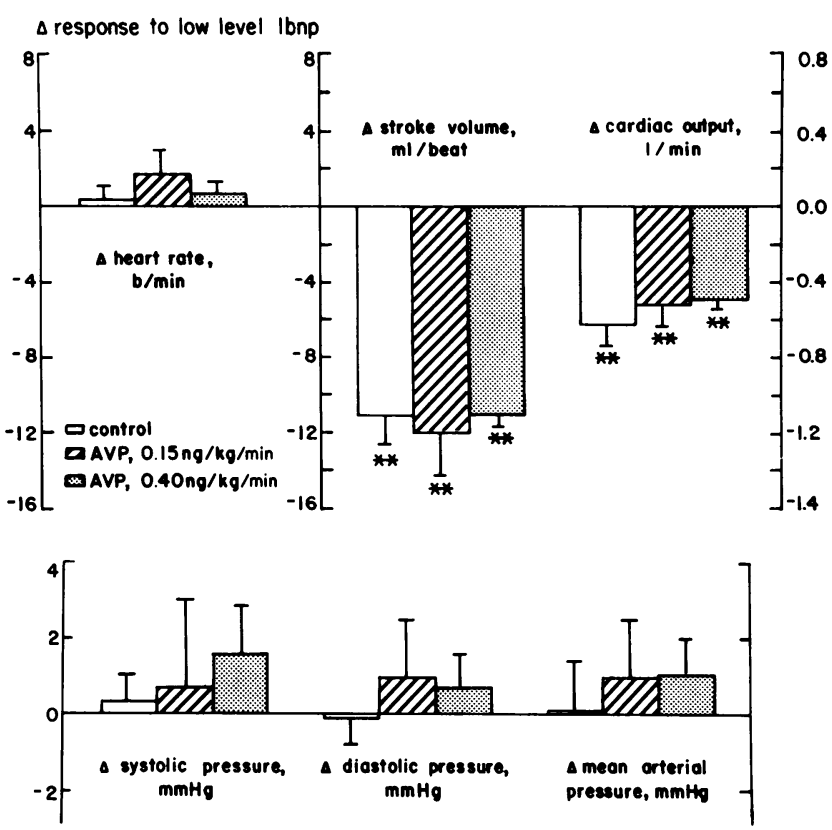

Figure 5. Responses of 10 volunteers to $10 \mathrm{mmHg}$ of lbnp. Heart rate and blood pressure were unchanged. Stroke volume and cardiac output declined significantly $\left({ }^{* *}, P<0.01\right)$; however, these declines were not different during AVP infusion compared with control (preinfusion) responses.

similar during control, low-, and high-dose AVP infusion periods. This suggests that the stimulus to low pressure baroreceptors was similar during control and during both AVP infusion periods.

In a previous study, Myers and Honig (37) demonstrated that vascular resistance changes are influenced by the initial vessel diameter and tone. Since AVP increased baseline total peripheral resistance (Fig. 4), it was possible that enhanced resistance responses might have been due to the increased baseline resistance. To minimize this potential bias, the relationship between the

$\Delta$ response to low level lbnp

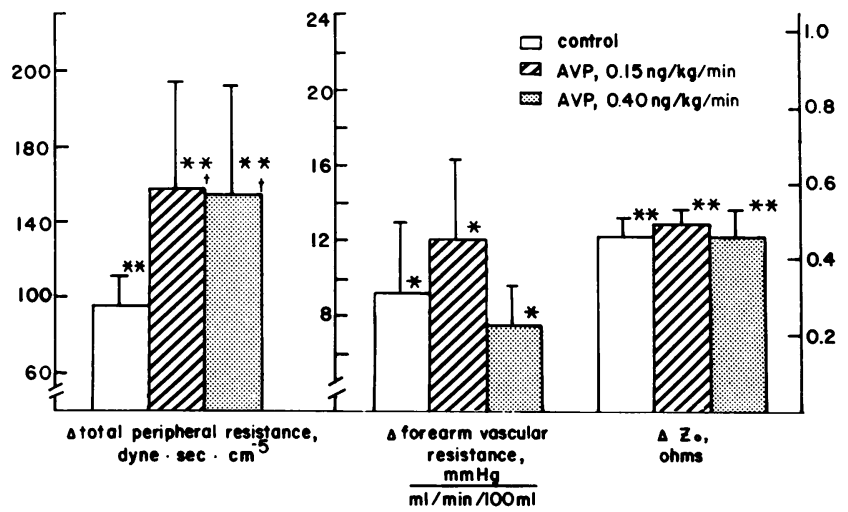

Figure 6. Responses of 10 volunteers to $10 \mathrm{mmHg}$ of lbnp. Resistance and Zo were significantly increased from pre-lbnp control $\left(^{*},{ }^{* *}, P\right.$ $<0.05$ and 0.01 ). Similar increases in Zo imply that thoracic blood volume decreases were similar. Total peripheral resistance responses were augmented during AVP infusion $(+, P<0.05)$ compared to control (pre-AVP) responses. control (pre-lbnp) total peripheral resistance for each subject was graphically related (linear regression) to the average change in total peripheral resistance produced by lbnp (Fig. 7). The steeper regression lines obtained during AVP infusion indicate a true enhancement of the resistance responses to lbnp rather than exaggerated responses related to the increased baseline resistance. Augmentation of reflex resistance responses by AVP infusion could not be attributed to changes in the reflex regulation of the forearm vascular bed. Forearm resistance responses to lbnp were not enhanced by AVP (Fig. 6).

\section{Discussion}

The influence of AVP on cardiovascular and autonomic reflex function has been identified in experimental animals $(1-5,33)$, but little is known about comparable effects of AVP in man. In the present study, infusions of two concentrations of AVP, which increased plasma levels an average of 13 and $30 \mathrm{pg} / \mathrm{ml}$, respectively, decreased heart rate and cardiac output and produced dose-dependent increases of total peripheral and forearm vascular resistances. The increases in resistance were associated with virtually no increases of blood pressure because of offsetting reductions in cardiac output. This phenomenon was first observed by Montani et al. (2) in conscious dogs, and in that species, it appeared to be primarily dependent on arterial baroreceptor reflexes as the AVP-induced fall in cardiac output was greatly attenuated following sino-aortic baroreceptor denervation. Furthermore, in baroreceptor-denervated dogs and in patients with autonomic dysfunction $(2,3,7,8)$, small elevations of plasma AVP similar to those in this study resulted in $20-50 \mathrm{mmHg}$ increases in mean arterial pressure.

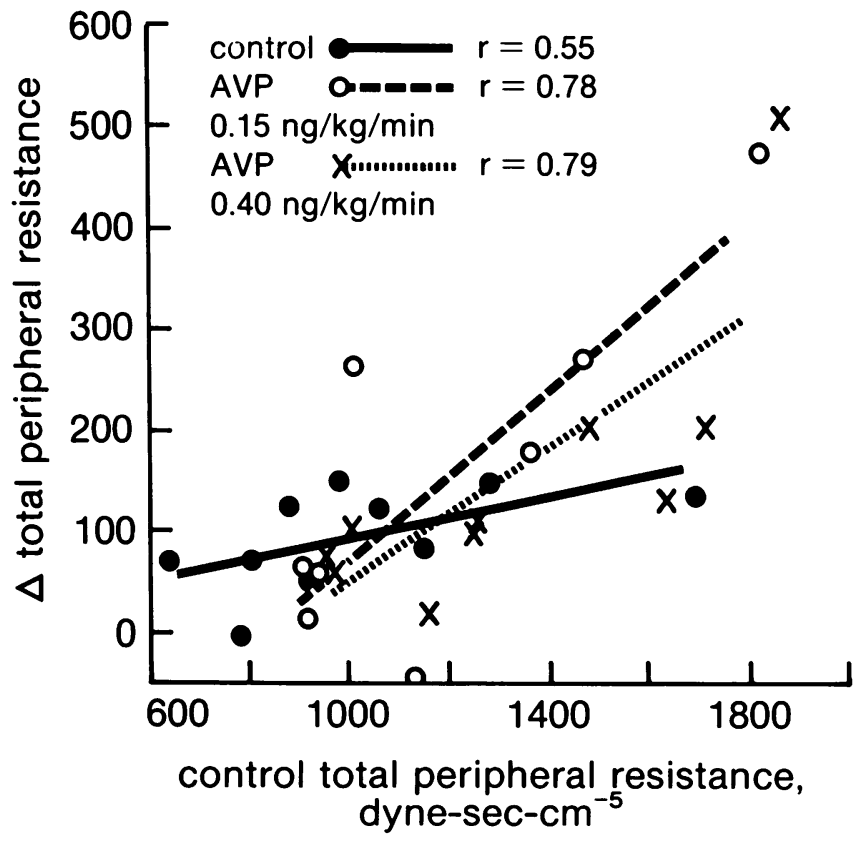

Figure 7. Individual control (pre-lbnp) total peripheral resistance responses (abscissa) and their respective change in total peripheral resistance produced by lbnp (ordinate). The steeper regression lines during AVP infusion indicate that reflex resistance responses were augmented (analysis of co-variance, $P<0.05$ ). 
AVP action on cardiac function and peripheral resistance. The mechanisms whereby AVP lowered heart rate and cardiac output were not ascertained in the present study. Data from anesthetized animals have suggested that high pharmacologic doses of AVP can influence the heart by direct effects on the sino-aortic node and/or direct coronary vasoconstriction with an associated decrease of contractility (38-40). At lower doses, similar to those used in this investigation, AVP increased vagal restraint on the heart by activation of the baroreflex $(39,40)$. AVP may also act centrally to increase vagal-cardiac efferent activity. Intravertebral infusions of AVP in conscious dogs were noted to produce greater declines in heart rate compared with intravenous infusions, despite similar elevations of plasma AVP levels (41). Furthermore, since sino-aortic baroreceptor denervation abolishes AVP-induced bradycardia in dogs, it has been suggested that the central effects of AVP are modulated by an afferent baroreceptor activity (42).

There are no previous studies in man which have examined the effects of small increases in plasma AVP on stroke volume. Impedance cardiography which reliably detects small changes in stroke volume, $(18,19,22-24)$ revealed a significant $8 \%$ reduction in stroke volume during small physiologic elevations in plasma AVP. This response is most likely not related to an increased afterload since arterial blood pressure was minimally elevated. A decrease in preload was also probably not involved since Zo, an index of thoracic blood volume, was unchanged during AVP infusions.

AVP has potent direct effects on vascular smooth muscle. The concentration of AVP required to decrease rat mesenteric arteriolar wall diameter is only $1 \mathrm{pg} / \mathrm{ml}$ (11). Similar effects have been shown in larger blood vessels (43). These direct effects appear to explain the dose-dependent increase in total peripheral resistance and forearm vascular resistance during AVP infusion. It is likely that resistance increases are not due to increases of efferent sympathetic activity since AVP infusion decreases plasma norepinephrine (this study) and decreases lumbar sympathetic nerve activity in rabbits (5).

In this study, the cardiovascular effects of intravenous infusions of AVP were not compared with those produced by other substances. It has been shown that angiotensin II infusions in man produce increases in heart rate, blood pressure, and peripheral resistance with little change in cardiac output (44). This contrasts to our demonstration of bradycardia and virtually no pressor effects of AVP infusions.

Cardiopulmonary baroreflex function. Slight reductions in thoracic blood volume are sensed by cardiopulmonary baroreceptors on the low-pressure side of the circulation. Reflex responses consist of increases in forearm and splanchnic vascular resistance $(13,14)$. These responses are thought to be independent of arterial baroreflexes since heart rate and blood pressure are unchanged in man $(13,14)$. Furthermore, nonhypotensive hemorrhage in dogs activates cardiopulmonary baroreflexes without changing carotid sinus afferent traffic (45).

In the present study, the reduction in cardiopulmonary baroreceptor stimulation by lbnp was similar before and during AVP infusion, as seen by the similar increases in Zo (an index of decreases of thoracic blood volume). Associated with this decreased stimulus to cardiopulmonary baroreceptors were significant reflex increases of total peripheral resistance and forearm vascular resistance. The increases in total peripheral resistance were augmented during AVP infusion. Furthermore, enhanced total peripheral resistance responses were augmented similarly during both infusion doses of AVP, which suggests that this effect may be maximal at plasma AVP concentrations only slightly above baseline and below $20 \mathrm{pg} / \mathrm{ml}$.

Augmented total peripheral resistance responses to lbnp did not appear to be due to a reflex enhancement of forearm vascular resistance since significant potentiation of responses by AVP were not seen in this bed. One study in anesthetized cats demonstrated a preferential effect of AVP on augmenting reflex vasoconstriction of intestinal vessels during hemorrhage (48). It is possible that the reflex regulation of this vascular bed may be preferentially enhanced by vasopressin during lbnp. Furthermore, AVP may augment vasoconstriction by its reported effect on sympathetic ganglion, nerve terminals, and adrenergic receptors $(11,12,46,47)$.

A similar enhancing effect of AVP on resistance increases provoked by carotid baroreceptor hypotension in dogs has been observed by Cowley et al. (4). This response may be related to a direct effect of AVP on carotid arterial vessels (43). In anesthetized rabbits, increases in single fiber and whole nerve afferent traffic in response to carotid sinus pressure changes are augmented by AVP infusion (49).

In this study, it is likely that arterial baroreceptors were not involved in the exaggerated resistance response to lbnp during AVP infusion. Heart rate was unchanged by lbnp both before and after vasopressin infusion, which suggests that arterial baroreflexes were not activated.

Angiotensin II can influence cardiovascular control mechanisms in man (50) and alter responses to AVP infusion in rats (35). Therefore, captopril was given to prevent changes in plasma angiotensin II which may have occurred during AVP infusion or lbnp exposure. The elevation of plasma renin activity was used as an index of the effectiveness of the blockade of angiotensin converting enzyme. A previous study demonstrated that pressor responses to exogenous angiotensin I are inhibited for more than $4 \mathrm{~h}$ after smaller doses of captopril (51). Thus it is unlikely that changes in plasma angiotensin II influenced responses ascribed to AVP.

Captopril is also known to increase plasma bradykinin and prostaglandin $E_{2}$ levels (52). If these influenced cardiovascular responses, their effect should have been constant during control and during each period of AVP infusion.

Our results indicate that physiologic levels of AVP have a significant effect on human cardiovascular function and autonomic reflex mechanisms. AVP decreased heart rate and stroke volume. This response was most likely due to changes in vagalcardiac activity and possibly due to direct effects of AVP on the heart. Pressor responses to AVP infusion were buffered by the decline in cardiac output. The direct vasoconstrictor effects of AVP on vascular smooth muscle resulted in progressive increases in total peripheral resistance and forearm vascular resistance during infusions of AVP.

Reflex increases of total peripheral resistance elicited by reductions in cardiopulmonary baroreceptor stimuli were augmented during AVP infusion. This effect may have been due to potentiation of splanchnic resistance responses to lbnp during AVP infusion, since increases in forearm vascular resistance were not enhanced. AVP augmentation of reflex resistance responses may have an important physiologic role. The results of this study imply that the release of AVP during hemorrhage or orthostasis may contribute importantly to the maintenance of arterial pres- 
sure, both by direct vasoconstriction and by augmenting reflex vasoconstriction mediated by cardiopulmonary baroreceptors in man.

\section{Acknowledgments}

We thank Anna Stadnicka for her careful preparation of blood samples during each study and her assistance in data reduction. We also thank Ms. Deborah Schoenborn and Ms. Sharon Taylor for their careful analysis of plasma hormones and Jean Ryland for her secretarial assistance.

\section{References}

1. Szczepanska-Sadowska, E. 1973. Hemodynamic effects of a moderate increase of the plasma vasopressin level in conscious dogs. Pfluegers Arch. Eur. J. Physiol. 338:313-322.

2. Montani, J. P., J. F. Liard, J. Schoun, and J. Mohring. 1980. Hemodynamic effects of exogenous and endogenous vasopressin at low plasma concentrations in conscious dogs. Circ. Res. 47:346-355.

3. Cowley, A. W. Jr., E. Monos, and A. C. Guyton. 1974. Interaction of vasopressin and the baroreceptor reflex system in the regulation of arterial blood pressure in the dog. Circ. Res. 34:505-514.

4. Cowley, A. W. Jr., D. Merrill, J. Osborn, and B. J. Barber. 1984. Influence of vasopressin and angiotensin on baroreflexes in the dog. Circ. Res. 54:163-172.

5. Schmid, P. G., F. M. Sharabi, G. B. Guo, F. M. Abboud, and M. D. Thames. 1984. Vasopressin and oxytocin in the neural control of the circulation. Fed. Proc. 43:97-102.

6. Khokhar, A. M., J. D. H. Slater, J. Ma, and C. M. Ramage. 1980. The cardiovascular effect of vasopressin in relation to its plasma concentration in man and its relevance to high blood pressure. Clin. Endocrinol. 13:259-266.

7. Mohring, J., K. Glanzer, J. A. Maciel Jr., R. Dusing, H. J. Kramer, R. Arbogast, and J. Koch-Weser. 1980. Greatly enhanced pressor response to antidiuretic hormone in patients with impaired cardiovascular reflexes due to idiopathic orthostatic hypotension. J. Cardiovasc. Pharmacol. 2: 367-376.

8. Wagner, H. N. Jr., and E. Braunwald. 1956. The pressor effect of the antidiuretic principle of the posterior pituitary in orthostatic hypotension. J. Clin. Invest. 35:1412-1418.

9. Leon, D. F., J. A. Shaver, and J. J. Leonard. 1970. Reflex heart rate control in man. Am. Heart J. 80:729-739.

10. Scher, A. M., W. W. Ohm, K. Bumgarner, R. Boynton, and A. C. Young. 1972. Sympathetic and parasympathetic control of heart rate in the dog, baboon and man. Fed. Proc. 31:1219-1225.

11. Altura, B. M., and B. T. Altura. 1977. Vascular smooth muscle and neurohypophyseal hormones. Fed. Proc. 36:1853-1860.

12. Bartelstone, H. J., and P. A. Nasmyth. 1965. Vasopressin potentiation of catecholamine actions in dog, rat, cat, and rat aortic strip. Am. J. Physiol. 208:754-762.

13. Ebert, T. J. 1983. Carotid baroreceptor reflex regulation of forearm vascular resistance in man. J. Physiol. (Lond.) 337:655-664.

14. Johnson, J. M., L. B. Rowell, M. Niederberger, and M. M. Eisman. 1974. Human splanchnic and forearm vasoconstrictor responses to reduction of right atrial and aortic pressure. Circ. Res. 34:515-524.

15. Segar, W. E., and W. W. Moore. 1968. The regulation of antidiuretic hormone release in man. J. Clin. Invest. 47:2143-2151.

16. Davies, R., and M. L. Forsling. 1975. Vasopressin release after postural changes and syncope. J. Endocrinol. 65:59-60P.

17. Witney, R. J. 1953. The measurement of volume changes in human limbs. J. Physiol. (Lond.). 121:1-27.

18. Ebert, T. J., D. L. Eckberg, G. M. Vetrovec, and M. J. Cowley. 1984. Impedance cardiograms reliably estimate beat-by-beat changes of left ventricular stroke volume in humans. Cardiovasc. Res. 18:354-360.

19. Smith, J. J., J. E. Bush, V. T. Wiedmeier, and F. E. Tristani.
1970. Application of impedance cardiography to study of postural stress. J. Appl. Physiol. 29:133-7.

20. Lababidi, Z., D. A. Ehmke, R. E. Durnin, P. E. Leaverton, and R. M. Lauer. 1971. Evaluation of impedance cardiac output in children. Pediatrics. 47:870-9.

21. Naggar, C. Z., D. B. Dobnik, A. P. Flessas, B. J. Kripke, and T. J. Ryan. 1975. Accuracy of the stroke index as determined by the transthoracic electrical impedance method. Anesthesiology. 42:201-5.

22. Baker, L. E., W. V. Judy, L. E. Geddes, F. M. Langley, and D. W. Hill. 1971. The measurement of cardiac output by means of electrical impedance. Cardiovasc. Res. Cent. Bull. 9:135-45.

23. Denniston, J. C., J. T. Maher, J. T. Reeves, J. C. Cruz, A. Cymerman, and R. F. Grover. 1976. Measurement of cardiac output by electrical impedance at rest and during exercise. J. Appl. Physiol. 40:91-5.

24. Gabriel, S., J. H. Atterhog, L. Oro, and L. G. Ekelund. 1976. Measurement of cardiac output by impedance cardiography in patients with myocardial infarction. Scand. J. Clin. Lab. Invest. 36:29-34.

25. Boer, P., J. C. Roos, G. G. Geyskes, and E. J. Dorhout Mees. 1979. Measurement of cardiac output by impedance cardiography under various conditions. Am. J. Physiol.: Heart Circ. Physiol. 6:H491-6.

26. Secher, N. J., A. Thomsen, and P. Amsbo. 1977. Measurement of rapid changes in cardiac stroke volume. An evaluation of the impedance cardiography method. Acta Anaesthesiol. Scan. 21:353-8.

27. Grossman, S. H., D. Davis, J. C. Gunnells, and D. G. Shand. 1982. Plasma norepinephrine in the evaluation of baroreceptor function in humans. Hypertension (Dallas). 4:566-571.

28. Leupker, R. V., J. R. Michael, and J. R. Warbasse. 1973. Transthoracic electrical impedance: quantitative measure of thoracic fluid volume. Am. Heart J. 85:83-93.

29. Patterson, R. P., W. G. Kubicek, D. A. Witsoe, and A. H. L. From. 1978. Studies on the effect of controlled volume change on the thoracic electrical impedance. Med. Biol. Eng. Comput. 16:531-536.

30. Hill, D. W., S. N. Mohapatra, K. C. Welham, and M. L. Stevenson. 1976. The effect of progressive decrease in the circulating blood volume of the dog on the transthoracic impedance. Europ. J. Intens. Care Med. 2:119-124.

31. Ebert, T. J., J. J. Smith, J. A. Barney, D. C. Merrill, and G. K. Smith. 1986. The use of thoracic impedance for determining thoracic blood volume changes in man. Aviat. Space Environ. Med. 57:49-53.

32. Sealey, J. E., J. Gorten-Banes, and J. H. Laragh. 1972. The renin system: variations in man measured by radioimmunoassay or bioassay. Kidney Int. 1:240-253.

33. Cowley, A. W., S. Switzer, and M. Guinn. 1980. Evidence and quantification of the vasopressin arterial pressure control system in dogs. Circ. Res. 46:58-67.

34. Cowley, A. W., Jr., W. C. Cushman, E. W. Quillen Jr., M. M. Skelton, and H. G. Langford. 1981. Vasopressin elevation in essential hypertension and increased responsiveness to sodium intake. Hypertension (Dallas). 3:I-93-I-100.

35. McNeil, J. R., W. C. Wilcox, and C. C. Y. Pang. 1977. Vasopressin and angiotensin: reciprocal mechanisms controlling mesenteric conductance. Am. J. Physiol. 232:H260-H266.

36. Spertini, F., H. R. Brunner, B. Waeber, and H. Gavras. 1981. The opposing effects of chronic angiotensin-converting enzyme blockade by captopril on the responses to exogenous angiotensin II and vasopressin vs. norepinephrine in rats. Circ. Res. 48:612-618.

37. Myers, H. A., and C. R. Honig. 1969. Influence of initial resistance on magnitude of response to vasomotor stimuli. Am. J. Physiol. 216: 1429-1436.

38. Heyndrickx, G. R., D. H. Boettcher, and S. F. Vatner. 1976. Effects of angiotensin, vasopressin, and methoxamine on cardiac function and blood flow distribution in conscious dogs. Am. J. Physiol. 231:15791587.

39. Varma, S., B. P. Jaju, and K. P. Bhargava. 1969. Mechanism of vasopressin-induced bradycardia in dogs. Circ. Res. 24:787-792. 
40. Nakano, J. 1967. Studies on the cardiovascular effects of synthetic vasopressin. J. Pharmacol. Exp. Ther. 157:19-31.

41. Liard, J. F., O. Deriaz, M. Tschopp, and J. Schoun. 1981. Cardiovascular effects of vasopressin infused into the vertebral circulation of conscious dogs. Clin. Sci. (Lond.). 61:345-347.

42. Cowley, A. W. Jr. 1982. Vasopressin and cardiovascular regulation. Int. Rev. Physiol. 26:189-242.

43. Monos, E., R. H. Cox, and L. H. Peterson. 1978. Direct effect of physiological doses of arginine vasopressin on the arterial wall in vivo. Am. J. Physiol. 234:H167-H172.

44. Scropp, G. C., and R. D. Lowe. 1968. Central pressor effect of angiotensin mediated by the parasympathetic nervous system. Nature (Lond.). 220:1331-1332.

45. Thames, M. D., M. Jarecki, and D. E. Donald. 1978. Neural control of renin secretion in anesthetized dogs: interactions of cardiopulmonary and carotid baroreceptors. Cir. Res. 42:237-245.

46. Commarato, M. A., and B. K. B. Lum. 1969. Cardiovascular interactions of amphetamine and ephedrine with norepinephrine and with vasopressin. Eur. J. Pharmacol. 7:125-134.
47. Imaizumi, T., and M. D. Thames. 1986. Influence of intravenous and intracerebroventricular vasopressin on baroreflex control of renal nerve traffic. Circ. Res. In press.

48. McNeill, J. R., R. D. Stark, and C. V. Greenway. 1970. Intestinal vasoconstriction after hemorrhage: roles of vasopressin and angiotensin. Am. J. Physiol. 219:1342-1347.

49. Holmes, A. E., and J. R. Ledsome. 1984. Effect of norepinephrine and vasopressin on carotid sinus baroreceptor activity in anesthetized rabbit. Experientia (Basel). 40:825-827.

50. Ebert, T. J. 1985. Captopril potentiates chronotropic baroreflex responses to carotid stimuli in man. Hypertension (Dallas). 7:602-606.

51. Ferguson, R. K., H. R. Brunner, G. A. Turnini, H. Gavras, and D. N. McKinstry. 1977. A specific orally active inhibitor of angiotensinconverting enzyme in man. Lancet. i:775-778.

52. Swartz, S. L., G. H. Williams, N. K. Hollenburg, L. Levine, R. G. Dluhy, and T. J. Moore. 1980. Captopril-induced changes in prostaglandin production; relationship to vascular responses in normal man. J. Clin. Invest. 65:1257-1264. 